\title{
A BOUNDARY MAXIMUM PRINCIPLE FOR DEGENERATE ELLIPTIC-PARABOLIC INEQUALITIES, FOR CHARACTERISTIC BOUNDARY POINTS
}

\author{
BY SALLY ELLENE MYERS ${ }^{1}$
}

Communicated by James Serrin, September 29, 1973

Let

$$
L u=\sum_{i, j=1}^{n} a_{i j}(x) \frac{\partial^{2} u}{\partial x_{i} \partial x_{j}}+\sum_{j=1}^{n} b_{i}(x) \frac{\partial u}{\partial x_{i}}, \quad a=\left(a_{i j}(x)\right) \geqq 0,
$$

be defined on an open set $\Omega \subset R^{n}$. We consider solutions $u=u(x) \in C^{2}(\Omega) \cap$ $C(\bar{\Omega})$ of $L u \geqq 0$ which attain their maximum value $M$ at $P$, a characteristic boundary point of $\Omega$.

Propagation SET. (See [3] and [1].) Let the diffusion vector field $\alpha^{k}(x)$ be the $k$ th column vector of the $n \times n$ matrix $\alpha(x)$, where $\alpha^{2}=a$, and let the drift vector field $\beta(x)$ be defined by $\beta_{i}=b_{i}-\sum_{j=1}^{n}\left(a_{i j}\right)_{x_{j}}$, $i=1, \cdots, n$. Assume that $\alpha^{1}, \alpha^{2}, \cdots, \alpha^{n}, \beta \in C^{1}(B)$, where $B \subseteq R^{n}$ is open and $\bar{\Omega} \subseteq B$. For $P \in \delta \Omega$, the propagation set $S(P, \Omega)$ is generated by segments of trajectories in $\Omega$ of vector fields $\lambda_{0} \beta+\lambda_{1} \alpha^{1}+\lambda_{2} \alpha^{2}+\cdots+$ $\lambda_{n} \alpha^{n}, \lambda_{0} \geqq 0$, where the scalar functions $\lambda_{k}=\lambda_{k}(x) \in C^{1}, k=0,1, \cdots, n$. $S(P, \Omega)$ is the closure of $S(P, \Omega)$ in $\Omega$.

Curvature Condition. Let $P \in \delta \Omega$ be a characteristic boundary point, that is, $v a(P) \nu=0$, where $v$ is the unit inner normal to $\delta \Omega$ at $P$. Then $\nu \alpha^{k}(P)=0$ for each $k, k=1, \cdots, n$. Let $\Pi^{k}$ be the plane of $\alpha^{k}(P)$ and $v$ through $P$. In this plane, the cross-section of $\delta \Omega$ and the projection of the trajectory of $\alpha^{k}$ through $P$ are curves which are perpendicular to $v$ at $P$. Let the curvatures of these curves at $P$ be $\tau_{k}$, for the sectional curvature of $\delta \Omega$, and $\sigma_{k}$, for the 'shadow curvature' [4] of the trajectory of $\alpha^{k}$. Finally, define the 'excess curvature' $\rho_{k}$ to be the difference $\tau_{k}-\sigma_{k}$.

Lemma. $\quad \beta v-\sum_{k=1}^{n} \rho_{k}\left|\alpha^{k}\right|^{2}=b v-\sum_{k=1}^{n} \tau_{k}\left|\alpha^{k}\right|^{2}$ at $P$. (The sums are over those $k$ for which $\alpha^{k}(P) \neq 0$.)

For the following results to hold, the curvature condition $\beta \nu-$ $\sum_{k=1}^{n} \rho_{k}\left|\alpha^{k}\right|^{2}>0$ must be satisfied at $P$.

AMS (MOS) subject classifications (1970). Primary 35J25, 35J70, 35K20.

Key words and phrases. Elliptic-parabolic operators, maximum principle.

${ }^{1}$ This note summarizes results contained in the author's Ph.D. thesis, which was written under the direction of Professor Raymond Redheffer at the University of California, Los Angeles. 
Theorems. Theorem 1 below generalizes a result of Friedman [2, Theorem 1] for parabolic inequalities. In addition to generalizing Friedman's curvature condition, we assume that the solution of $L u \geqq 0$ is of class $C^{2}$ near $P$, and thereby can dispense with his other conditions.

THEOREM 1. Let $P \in \delta \Omega$ be a characteristic boundary point and let $\beta \nu-\sum_{k=1}^{n} \rho_{k}\left|\alpha^{k}\right|^{2}>0$ hold at $P$. If $u(P)=M \geqq u(x)$ for all $x \in S(P, \Omega)$, if $L u \geqq 0$ on $S(P, \Omega)$, and if $u \in C^{2}$ near $P$, then (a) $D_{0} u(P)=D_{1} u(P)=$ $D_{2} u(P)=\cdots=D_{n} u(P)=0$ and $\sum_{k=1}^{n} D_{k} D_{k} u(P)=0$, and $(\mathrm{b})$ there exists $a$ point $Q$ in $\Omega$ such that $u(Q)=M$ and $Q \in \bar{S}(P, \Omega)$. (Here $D_{0}\left(D_{k}\right)$ denotes differentiation along a drift (diffusion) trajectory, and $D_{k} D_{k} u$ is the second derivative along the kth diffusion trajectory.)

THEOREM 2. If the above hypotheses are strengthened to $u(P)=M \geqq$ $u(x)$ for all $x \in \Omega$ and $L u \geqq 0$ in $\Omega$, then to conclusion (a) one may add that $D_{\tau} u(P)=0$ for any direction $\tau$ which points into $\Omega$, that is, such that $\tau v>0$.

Counterexamples show that the curvature condition is best possible. That is, if $\beta v-\sum_{k=1}^{n} \rho_{k}\left|\alpha^{k}\right|^{2} \leqq 0$, the theorems need not hold.

Corollary. If $P \in \Omega$ is in a level surface of $u$, a solution of $L u=0$ in $S(P, \Omega)$, and the normal $\nu$ to this surface at $P$ is defined and satisfies $v a(P) \nu=$ 0 , then $\beta \nu=\sum_{k=1}^{n} \rho_{k}\left|\alpha^{k}\right|^{2}$ at $P$, where the excess curvatures $\rho_{k}$ are calculated using the sectional curvatures $\tau_{k}$ of the level surface.

When the assumption that $u$ is of class $C^{2}$ near $P$ is dropped, it can only be shown that if $u<M$ near $P$, then $D_{\tau} u(P)<0$ when $\tau$ points into $\Omega$. This may appear to violate the previous results, which show that $D_{\tau} u=0$. However, the previous theorems show that if $u$ is $C^{2}$ near $P$, then it is impossible to satisfy the hypothesis that $u<M$ near $P$.

THEOREM 3. Let $P \in \delta \Omega$ be a characteristic boundary point and let $\beta v-\sum_{k=1}^{n} \rho_{k}\left|\alpha^{k}\right|^{2}>0$ hold at $P$. If $u(P)=M>u(x)$ for all $x \in \bar{S}(P, \Omega)$, and if $L u \geqq 0$ on $S(P, \Omega)$, then $D_{\tau} u(P)<0$ for any $\tau$ which points into $S(P, \Omega)$ and into $\Omega$.

THEOREM 4. If the hypotheses of the last theorem are strengthened to $u(P)=M>u(x)$ for all $x \in \Omega$ and $L u \geqq 0$ in $\Omega$, then the conclusion is that $D_{\tau} u(P)<0$ for any $\tau$ which points into $\Omega$.

In his 1970 paper, Hill [3] presented two theorems (2 and 2') of the form of Theorems 3 and 4 above. As shown by counterexamples, Hill's theorems are incorrect for characteristic boundary points because he does not include the curvature condition. These examples also show that that curvature condition is best possible in Theorems 3 and 4 .

Examples. The two following examples ${ }^{2}$ illustrate the content of the above theorems.

\footnotetext{
${ }^{2}$ Suggested by Professor James Ralston.
} 
EXAMPLE 1. Let $\Omega=\left\{1<x^{2}+y^{2}<4\right\} \subseteq R^{2}$ and let $L u=u_{x x}+x^{2} u_{y y}$. Then the points $(0, \pm 1)$ and $(0, \pm 2)$ are characteristic boundary points. For each one of these points $P, S(P, \Omega)=\Omega$. At $P=(0, \pm 1), b v-$ $\tau_{1}\left|\alpha^{1}\right|^{2}=(0,0)(0, \pm 1)-(-1)(1)=1>0$. Let $u$ be a function that satisfies $L u \geqq 0$ on $\Omega$, and that attains its maximum value, $M$, on $\Omega$ at $P$. By Theorem 1 , if $u$ is of class $C^{2}$ near $P$, then $u$ must attain its maximum value at an interior point of $\Omega$. Therefore, (by Redheffer [4, Theorem 2]) $u$ is constant throughout $\Omega$. Thus, if $u$ were not constant, it could not be of class $C^{2}$ near $P$. Such a solution would have negative inward derivatives at $P$, by Theorem 4 applied to $\Omega^{*}=\Omega \cap N$, where $N$ is a neighborhood of $P$ such that $u<M$ in $\Omega^{*}$. At $P=(0, \pm 2), b v-\tau_{1}\left|\alpha^{1}\right|^{2}=(0,0)(0, \mp 1)-$ $\left(\frac{1}{2}\right)(1)=-\frac{1}{2}<0$, and the theorems yield no information.

EXAmPLE 2. For $\Omega=\left\{\frac{1}{4}<x^{2}+y^{2}<4\right\}$ and $L u=u_{x x}-u_{y}$, the characteristic boundary points of $\Omega$ are $\left(0, \pm \frac{1}{2}\right)$ and $(0, \pm 2)$. When $P=(0,2)$, $S(P, \Omega)=\Omega$. At this point, $b v-\tau_{1}\left|\alpha^{1}\right|^{2}=(0,-1)(0,-1)-\left(\frac{1}{2}\right)(1)=\frac{1}{2}>0$. Suppose that $u$ satisfies $L u \geqq 0$ on $\Omega$, and attains its maximum value $M$ on $\Omega$ at $(0,2)$. If $u$ is of class $C^{2}$ in a neighborhood of $P$, then let $N_{i}, \quad i=1,2, \cdots$, be neighborhoods of $P$ such that $\bigcap_{i=1}^{\infty} N_{i}=P$. By Theorem 1 applied to the sets $\Omega_{i}=\Omega \cap N_{i}$, there is a sequence of points $\left\{Q_{i}\right\}$ in $\Omega$ which converges to $P$ and such that $u\left(Q_{i}\right)=M, i=1,2, \cdots$. Thus, by Nirenberg's maximum principle for parabolic inequalities, $u \equiv M$ on $\Omega$. Therefore, if $u$ were not constant on $\Omega$, then it would not be of class $C^{2}$ near $P$, and $u$ would also satisfy the hypotheses of Theorem 4 near $P$. The inward derivatives of $u$ at $P$ would be negative.

If $P=\left(0, \frac{1}{2}\right)$, then $S(P, \Omega)=\Omega \cap\left\{y \leqq \frac{1}{2}\right\}$ and $b v-\tau_{1}\left|\alpha^{1}\right|^{2}=(0,-1)(0,1)-$ $(-2)(1)=1>0$. Consider a solution of $L u \geqq 0$ on $S(P, \Omega)$ which attains its maximum value $M$ relative to this set at $P$. If $u$ is of class $C^{2}$ near $P$, then Theorem 1 yields a sequence of points $\left\{Q_{i}\right\}$ in $\bar{S}(P, \Omega)$ which converges to $P$ and for which $u\left(Q_{i}\right)=M$. The points $Q_{i}, i=1,2, \cdots$, may all lie to one side of $P$. Thus, Nirenberg's theorem shows that $u=M$ either on $\bar{S}(P, \Omega) \cap\left(\left\{y \leqq-\frac{1}{2}\right\} \cup\{x>0\}\right)$ or on $\bar{S}(P, \Omega) \cap\left(\left\{y \leqq-\frac{1}{2}\right\} \cup\{x<0\}\right)$ ! If $u$ were not constant on either set, then it could not be of class $C^{2}$ near $P$. Theorem 3 yields no result in this case because no direction points into $\Omega$ and $S(P, \Omega)$ at $P$. However, if $u$ satisfied $L u \geqq 0$ on $\Omega$ and if $u<M$ held in $\Omega$ near $P$, then Theorem 4 would imply that the inward derivatives of $u$ at $P$ were negative.

The theorems can be applied at $\left(0,-\frac{1}{2}\right)$, but not at $(0,-2)$ where the curvature condition is not satisfied and $S(P, \Omega)$ is empty.

\section{REFERENCES}

1. J.-M. Bony, Principe du maximum, inégalité de Harnack et unicité du problème de Cauchy pour les opérateurs elliptiques dégénérés, Ann. Inst. Fourier (Grenoble) 19 (1969), fasc. 1, 277-304. MR 41 \#7486. 
2. A. Friedman, Remarks on the maximum principle for parabolic equations and its applications, Pacific J. Math. 8 (1958), 201-211. MR 21 \#1444.

3. C. D. Hill, $A$ sharp maximum principle for degenerate elliptic-parabolic equations, Indiana Univ. Math. J. 20 (1970/71), 213-229. MR 44 \#4382.

4. R. M. Redheffer, The sharp maximum principle for nonlinear inequalities, Indiana Univ. Math. J. 21 (1971), 227-248.

Department of Mathematics, University of Southern California, Los ANGeles, California 90007 